\title{
Development of Electrically Active Textiles
}

\author{
T Dias*, W Hurley*, R Monaragala* and R Wijeyesiriwardana** \\ *Textiles and Paper, School of Materials \\ The University of Manchester, UK \\ ** ReliSen LLC, Bentonville, Arkansas 72712 USA
}

\section{Introduction}

The current generation of textiles, including technical textiles are passive. However the next generation of textiles will have the ability to monitor its environment and interact accordingly in order to accomplish a pre-programmed functionality. Such textiles can be considered as truly smart textiles, and they would consist of three basic components:

1. sensing and measuring capability;

2. activation capability;

3. intelligence (programming capability).

One of the solutions for incorporating the above components into a textile structure is to create electrically active zones within the structure, whose electrical characteristics can change due to an environmental change or whose structural properties would change due to application of an electrical signal, for example change of dimensions due to the flow of an electrical current in the electrically active area. Generally textiles are made out of materials of very high electrical resistance and can, therefore, be considered as materials with good electrical insulating properties.

The aim of the paper is to demonstrate the development of electrically active knitted structures and switches which have resulted from the research carried out by my research group in the School of Materials of the University of Manchester, UK. Around eight years ago a multidisciplinary research group was set-up by me in order to develop electrically active textiles $\left(\mathrm{EAT}^{\circledR}\right)$; the skills set of the group comprised of knitting technology, electronics and software engineering and textile fibres.

Knitted structures exhibit following characteristics:

- good tensile recovery properties;

- superior drapability, which would provide excellent skin contact;

- breathability: the structures are air permeable, giving more comfort.

In view of the above we decided to utilise the latest knitting technology to develop EAT structures. Knitting technology provides several different production routes; circular and flat-bed weft knitting and warp knitting. One technology in particular, the modern computerised flat-bed knitting technology, has made quantum leaps during the last few decades thanks to the extensive use of mechatronic systems for all mechanical movements and CAD/CAM systems. The most important features of the technology are: 
- the precision positioning of fibres in 3D space; which is very useful in EAT construction, i.e. the placement of different fibres at appropriate positions in a knitted structure;

- the freedom to construct structures with different binding elements; which is useful for the optimisation of EAT for maximum sensitivity and performance;

- 3D structures add an additional dimension to the EAT construction;

- multilayer structures are very useful for constructing EAT with integrated power and data lines;

- true seamless garment knitting.

It is our belief that the best platform for the development of multilayered 3D EAT structures is provided by the above technology. Different layers can be used for creating arrays of sensors and conductive pathways for power and data lines. Such EAT structures would be washable, and the technology would enable them to be manufactured with a higher degree of precision at low cost (due to minimum labour involvement).

\section{Configuration of a knitted EAT}

The generic method of construction of a knitted EAT structure, which is capable of sensing and/or can be initiated to perform a pre-defined activity, is to knit a predetermined area of a knitted structure (base structure or substrate) with electro conductive fibres. The above area is defined as the 'Electro Conductive Ared' (ECA) in this text (Figure 1). The size, the shape and the method of fibre binding, i.e. the stitches, the tuck loops and the floats, and their organization within the base structure would determine the overall electrical characteristics of the ECA. One of the measurable electrical properties of a knitted EAT structure would be the electrical resistance/impedance of the ECA.

\section{Development of knitted heating structures}

A knitted structure that is capable of generating heat, when powered by an electrical current, was made by creating an ECA from an electroconductive yarn within a plain knitted structure produced from a nonconductive yarn such as Nylon 6-6. The electroconductive yarn was a silicone monofilament fibre loaded with carbon particles. A specific resistance of $10 \mathrm{~K} \Omega \mathrm{cm}^{-1}$ was measured at the monofilament

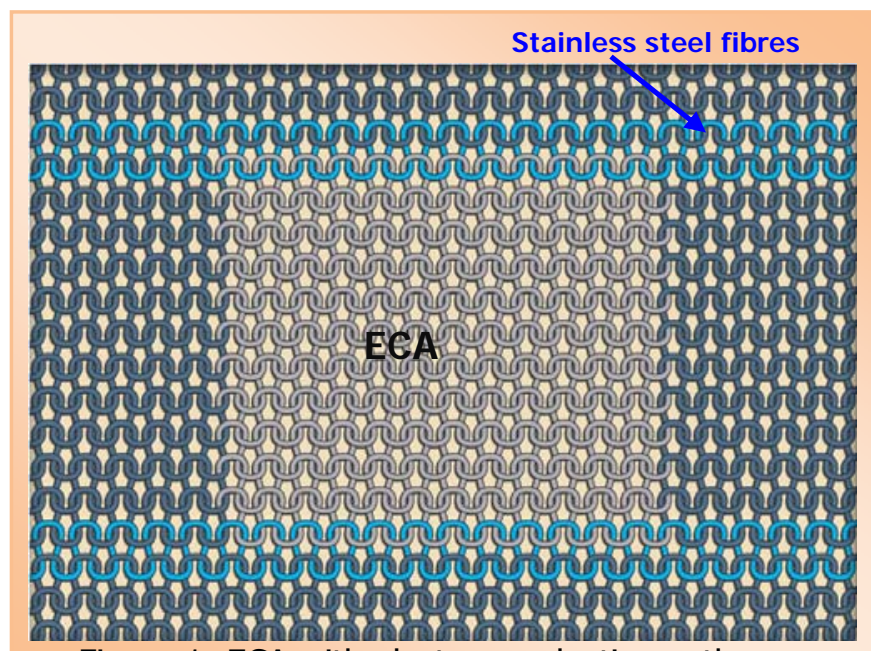

Figure 1: ECA with electro-conductive pathways yarn at its relaxed state. 
The EAT which is capable of generating heat was constructed by using electro conductive fibres of different conductivity levels and non conductive yarns. Stainless steel yarns $\left(10 \Omega \mathrm{cm}^{-1}\right)$ were used as the power lines of the structure and the ECA was created with the low/semi conductive carbon filled silicone monofilament $\left(10^{4} \Omega \mathrm{cm}^{-1}\right)$ described above. The base structure of the EAT was constructed from a plain knitted structure (Figure 1). The resistive model of the ECA was constructed by using the spatial orientation of the conductive fibres in the three dimensional space. Figure 1 highlights the electro conductive path of the ECA. The ECA comprises of three different regions knitted from different yarns, and hence it can be divided in to three different segments electrically. The first is the heating area (ECA), the second the powering elements and the third the base structure. In the following analysis, " $\mathbf{m}$ " is the number of courses (rows) and " $n$ " is the number of wales (columns) of the ECA (heating segment), which is knitted from the low conductive fibres (carbon loaded silicone monofilament).

\section{$\underline{\text { DC Equivalent Circuit }}$}

Stitch is the primary yarn binding element of a knitted structure, which is created when three yarn loops are interconnected together resulting in four yarn contact regions as shown in Figure $2 a$. The contact mechanics at the contact areas are very complex and their behaviour is not yet fully understood. If an incompressible and inextensible yarn (idealised yarn) is used then each contract region can be represented with two contact points (i.e. a total of eight contact points per stitch). However, for all standard yarns the yarn cross-over regions of a stitch can be represented as a line contact at the relaxed state shifting into an area contact as the knitted structure undergoes mechanical loading. Therefore the yarn cross-over

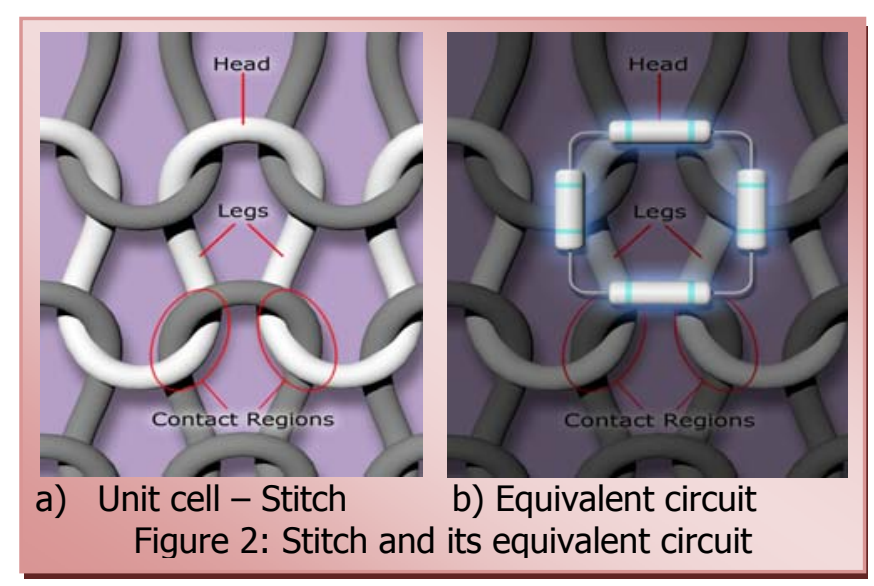

and four nodes (Figure $2 b$ ). regions of a stitch was considered as a short circuiting point for modelling the electrical equivalent circuit of the ECA, i.e. each yarn cross-over region of a stitch will act as a node in a resistive mesh. There are four yarn cross-over regions of a stitch; and, therefore, the DC equivalent circuit of a stitch was formulated using four resistances

The lengths $L_{\text {leg, }}$ and $L_{\text {head }}$ were calculated by considering the yarn path geometry in a unit cell of the ECA, which is a stitch. In knitting science the stitch geometry is defined with one half circle (stitch head), two straight lines (stitch legs) and two quarter circles (stitch feet). In a fully relaxed knitted structure (i.e. when the yarns constituting the stitches are in their minimum energy state) the length of yarn in a foot of a stitch would be half the length of yarn forming the head of the stitch. As such the DC equivalent circuit of the unit cell of the ECA was represented with four 
resistances; two resistances representing the heads $\left(\mathbf{R}_{\mathbf{H}}\right)$ and two resistances representing the two legs $\left(\mathbf{R}_{\mathbf{L}}\right)$ which are connected to each other as shown in Figure $2 b$. In the following analysis, " $m$ " is the number of courses (rows) and " $n$ " is the number of wales (columns) of the ECA, which is knitted from the low conductive fibres (e.g. carbon loaded silicone monofilament). In order to calculate the equivalent resistance of the ECA a resistor mesh was created with the four resistance model of the unit cell, as shown in Figure 3, from which the total equivalent resistance $\left(\boldsymbol{R}_{\mathbf{e q}}\right)$ was calculated for a defined powering configuration. The resistances of the unit cell will be influenced by the lengths of conductive yarn in the head/feet and the two legs of a stitch; these yarn lengths can be calculated under the assumption that in a fully relaxed

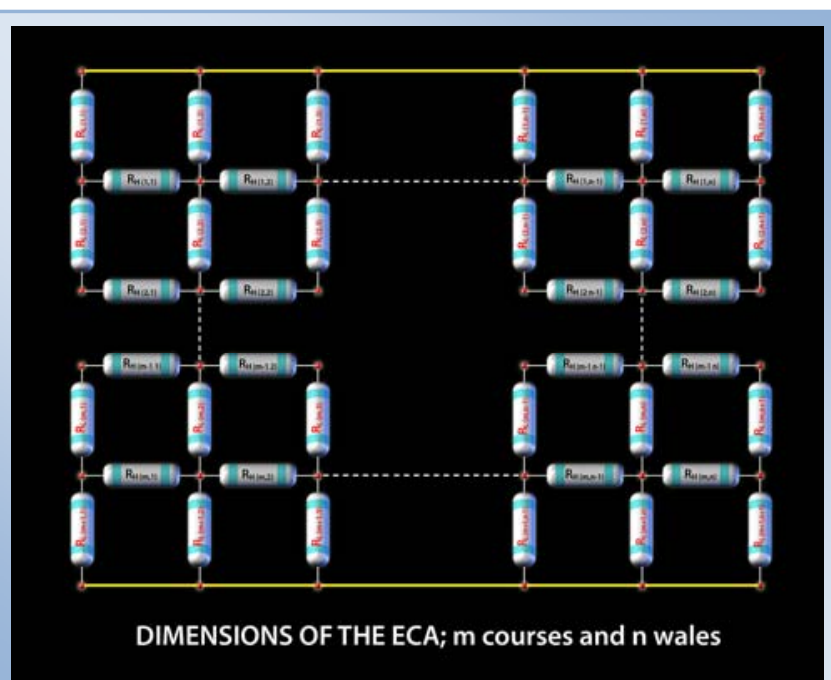

Figure 3: Resistor mesh model of the ECA knitted structure the geometry of the stitch head is defined by a half circle and the stitch leg by a straight line. The equivalent resistance of the ECA will depend on the total number of stitches constituting the ECA. The total number of stitches will, however, depend on the number of courses $(m)$ and the number of wales $(n)$ in the ECA. The equivalent resistance of an ECA of a given $m$ and $n$ will also depend on the length ratio between the $L_{\text {leg }}$ (length of yarn in the stitch leg) and the $L_{\text {head }}$ (length of yarn in the stitch head). The dependency of the equivalent resistance of an ECA $\left(R_{\text {eq }}\right)$ having $m$ courses and $n$ wales, when $L_{\text {leg }} / L_{\text {head }}=2$ is shown in the Figure 4.

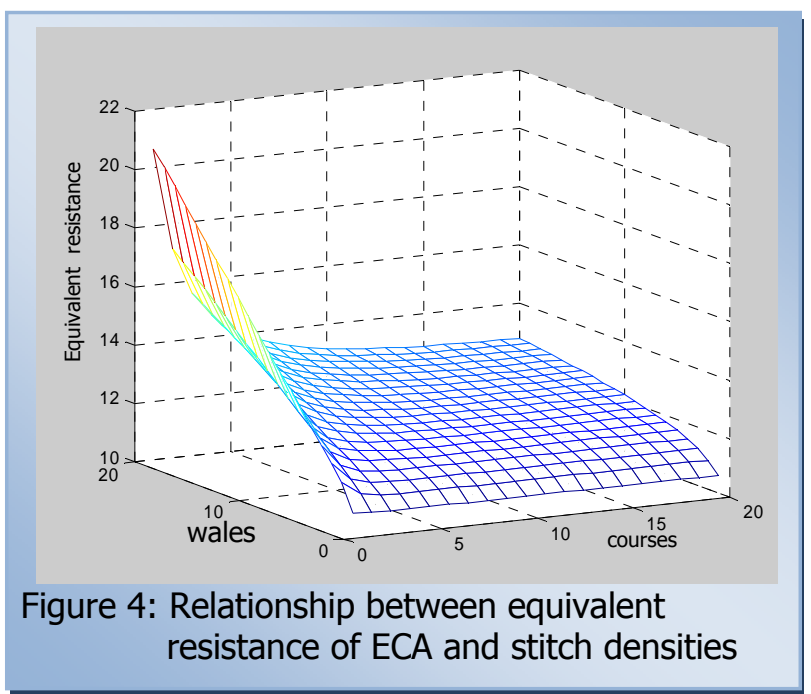

Our calculations show that for higher $L_{\text {leg }} / L_{\text {head }}$ ratio with constant $L_{\text {leg, }}$ the equivalent resistance of the $E C A, R_{\text {eq, }}$ would reduce. Figure 4 demonstrates how the number of courses and the number of wales would influence the equivalent resistance of the ECA. $R_{\text {eq }}$ would increase when the number of courses is increased, which is significant for a given number of wales. However an increase in the number of wales for an ECA with given number of courses would result in a reduction of the equivalent resistance of the ECA. The rate of reduction of the equivalent resistance of the ECA is significant within a limited range, and thereafter its rate of reduction becomes less significant and levels out. 
The power distribution of the stitches of the ECA will depend on the current distribution within the unit cells of the ECA, which will be determined by the resistances $R_{\text {head }}$ and $R_{\text {leg. }}$. Therefore, the current distribution in the ECA can be calculated from the mesh loop currents $\left(I_{m}\right)$ and the currents flowing in the stitch heads $\left(I_{H}\right)$ and legs $\left(I_{L}\right)$, which can be determined from:

$$
\mathrm{I}_{\mathrm{m}}=\mathrm{Z}_{\mathrm{m}}^{-1} \mathrm{~V}
$$

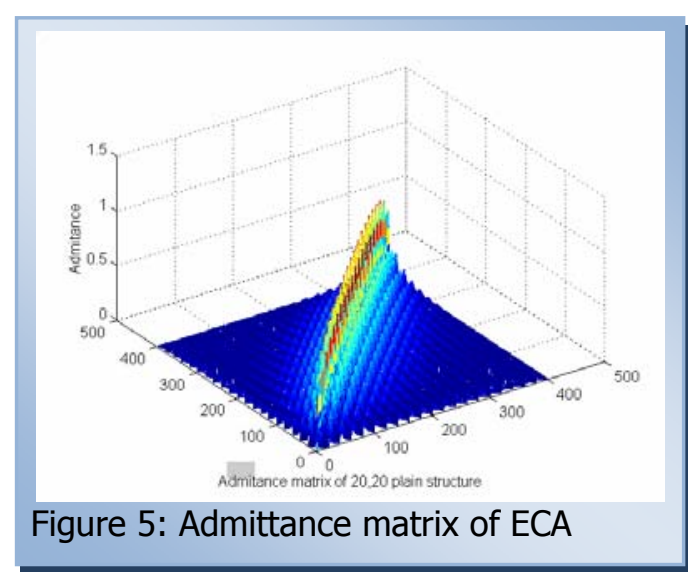

In the above equation $\mathrm{V}$ is the voltage applied to the ECA and $\mathrm{Z}_{\mathrm{m}}{ }^{-1}$ the admittance matrix of the ECA, which is given in Figure 5. For each stitch the distribution of the yarn in the stitch within the legs and the head of the stitch can be calculated (i.e. $L_{\text {leg }(i, j)}$ and $\left.L_{\text {head }(i, j)}\right)$ by considering the geometry of the yarn path in the stitch, and the corresponding resistances can be determined from the resistivity, $\mathrm{P}_{\mathrm{L}}$, and the cross-sectional area, $\mathrm{A}$, of the conductive

yarn using equation 2:

$$
R_{L(i, j)}=\frac{p_{L} L_{\text {Leg }(i, j)}}{A} \wedge R_{H(i, j)}=\frac{P_{L} L_{\text {Head }(i, j)}}{A}
$$

From equation (2) the resistance matrixes of stitch head, $R_{H}$, and stitch legs, $R_{L}$, can be obtained. By applying the electrical network theory of mesh analysis the equivalent resistance of the ECA, $R_{\text {eq, }}$, the voltage vector, $V_{M}$ and the current vector $I_{M}$ were determined. The necessary equations are given below.

$$
\begin{aligned}
& \mathrm{V}_{\mathrm{M}}=\{\mathrm{V}, 0,0,0, \ldots, 0\}_{\mathrm{m} * \mathrm{n}+1,1} \\
& \mathrm{I}_{\mathrm{M}}=\left\{\mathrm{I}_{0}, \mathrm{I}_{1,1}, \mathrm{I}_{1,2}, \ldots, \mathrm{I}_{2,1}, \ldots \mathrm{I}_{\mathrm{m}, \mathrm{n}}\right\}_{\mathrm{m} * \mathrm{n}+1,1} \\
& V_{M}=Z_{M} \times I_{M} \Rightarrow I_{M}=Z^{-1} M \times V_{M} \\
& \Rightarrow R_{e q}=\frac{1}{Z^{-1}{ }_{(1,1)}}-R_{0}
\end{aligned}
$$

Where $Z_{(1,1)}^{-1}$ is the $(1,1)$ element of $Z^{-1}{ }_{M}$. The distribution of current in the stitch heads I_ $H_{(m+1, n)}$ and the stitch legs I_L $L_{(m+1, n+1)}$ were calculated; with the above values the power distribution in the stitch heads $\left(P_{-} H\right)$ and the stitch legs $\left(P_{-} L\right)$ determined.

$$
\begin{aligned}
& P_{-} H_{(i, j)}=R_{H(i, j)} \times I_{-} H_{(i, j)}{ }^{2} \\
& P_{-} L_{(i, j)}=R_{L(i, j)} \times I \_L_{(i, j)}
\end{aligned}
$$

Under the assumption that any heat loss in the ECA is only due to radiation, the temperature distribution in the stitch heads $\left(T_{-} H\right)$ and stitch legs $\left(T_{-} L\right)$ were calculated. From the Plank's law of radiation;

$$
\begin{aligned}
& \left.T_{-} H_{(i, j)}=\left(P_{-} H_{(i, j)}\right) / K_{C}+T_{a}{ }^{4}\right)^{1 / 4} \\
& \left.T_{-} L_{(i, j)}=\left(P_{-} L_{(i, j)}\right) / K_{C}+T_{a}{ }^{4}\right)^{1 / 4}
\end{aligned}
$$


Where $K_{c}$ is radiation constant of the conductive fibre and the $T_{a}$ is the ambient temperature. The theoretical and measured results are given in the Figures 6 and 7; the temperature distribution within the ECA was measured by using pt100 thermocouples.

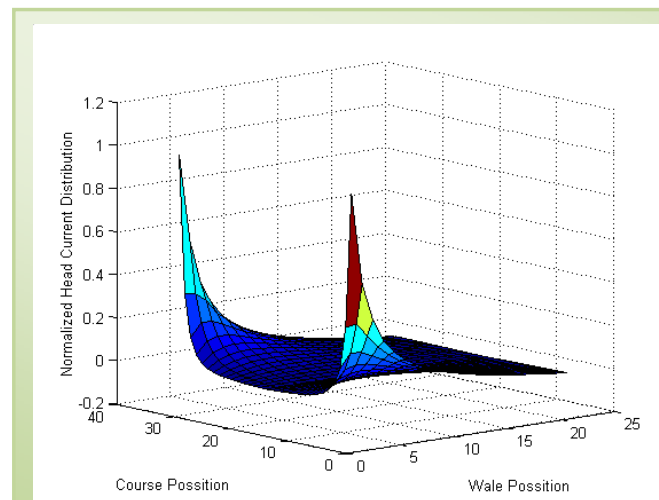

a) I_H distribution

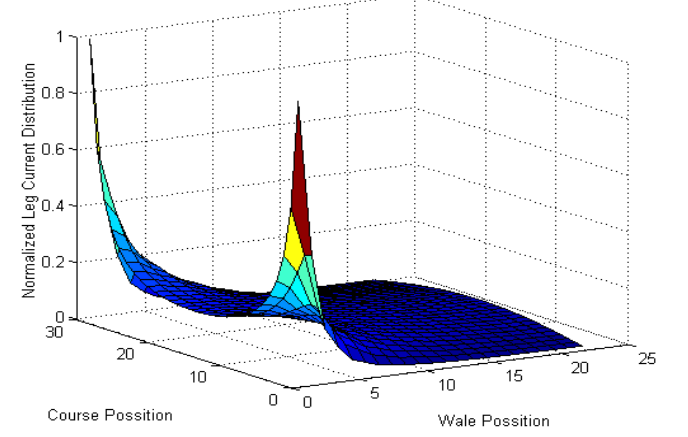

b) I_L distribution

Figure 6: Theoretical current flow in heads and legs of the stitches

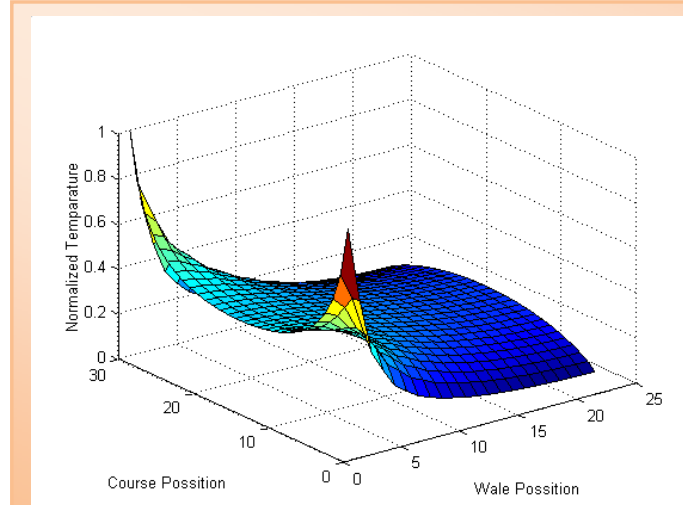

a) Stitch heads (T_H)

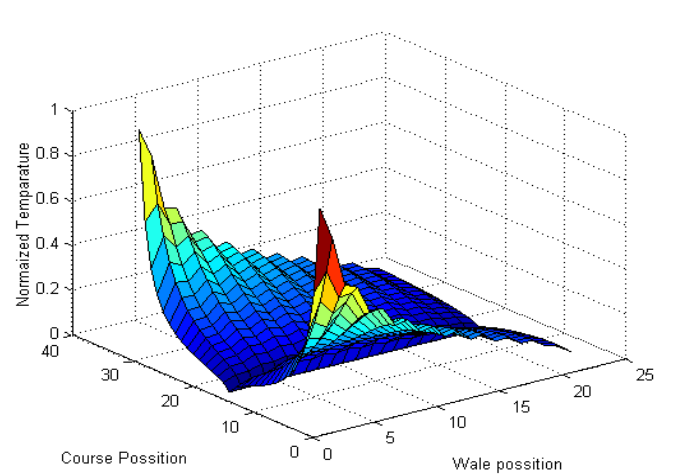

b) Stitch legs (T_L)

Figure 7: Normalised temperature distribution in stitches of ECA (theoretical)

\section{Development of Knitted Switches (K-Switch)}

Another application of ECAs is the development of knitted flexible switches, named as $\mathrm{K}$-Switches, which can easily be integrated into textile structures using modern flat-bed knitting technology. The principle of operation of the K-Switch is based upon the resistance change between two neighboring ECAs due to touching the ECAs with a human finger. Such a dual ECA switch consists of two or more conductive pathways which are isolated from each other by non-conductive yarns. The ECAs and the conductive pathways were created based on the concept of three colour jacquard using a computer flat-bed knitting machine. Yarn path notations for producing dual ECA switches are given in Figure 8. The conductive yarn was knitted on alternating needles on the back needle bed. It was only knitted on the front needle bed to create the ECA. Each conductive pathway was created with at least one course of conductive yarn followed by at least 2 courses of non-conductive yarn to insulate the pathway. The conductive yarn was knitted full width of the structure. 
In order to localise the switching behaviour to a specific visual reference region on the textile structure, the conductive pathways must be electrically insulated within the knitted structure with the exception of the switching area (dual ECA region). One elegant solution for insulating the conductive pathways was the use of jacquard knitting on both

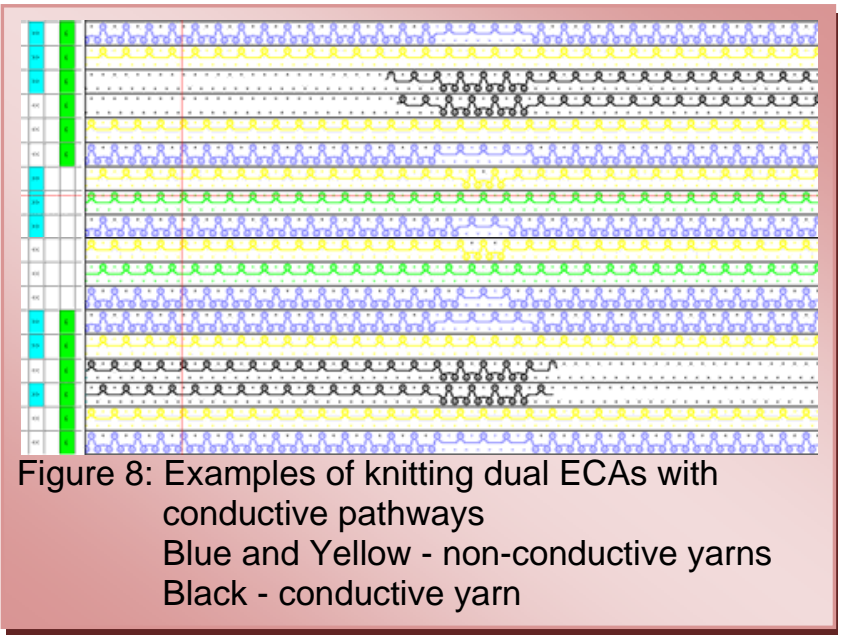
needle beds, which would enable the conductive pathways to be completely insulated from the technical front of the knitted structure. Another advantage of using electronic flat-bed knitting is the ability of creating pattern motifs to map the switches depending on the individual operation, see Figure 9.

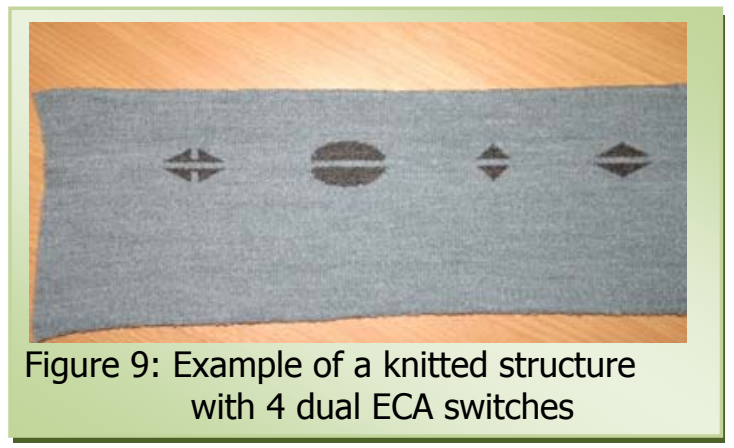

The electrical equivalent circuit of a dual ECA switch is given in Figure 10. A dc voltage of $12 \mathrm{~V}$ was applied between the conductive pathways of the two ECAs, and the potential at ECA2 with reference to the ground was measured. An open circuit

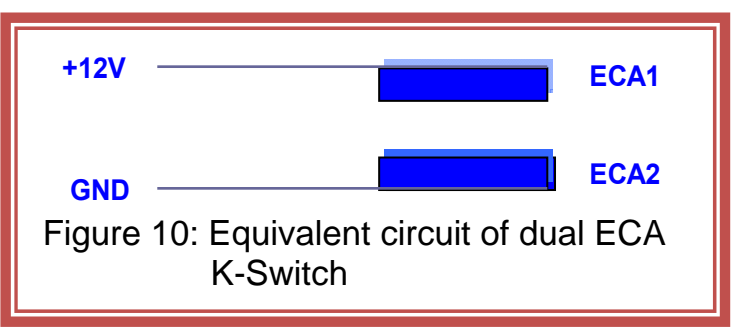
voltage of $5 \mathrm{mV}$ was measured, and when a gloved finger was placed on the two ECAs the voltage increased to 20 $\mathrm{mV}$; the value measured depended on the type of the glove. However when the dual ECA switch was touched by a bare finger a voltage greater than $7 \mathrm{~V}$ was observed. Based on the above data a comparator circuit was designed which can also be activated from a gloved finger.
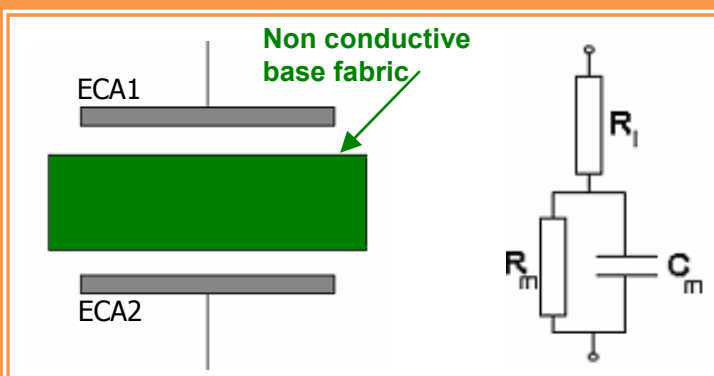

Figure 11: The impedance model of dual ECA K-Switch
A study of the ac performance of the dual ECA switches was also carried out. The impedance of the dual ECA switch was modeled using resistive and capacitive components. Figure 11 shows the impedance model of a dual ECA switch including conductive pathways. The impedance characteristics of the dual ECA switch was measured under open and closed 
circuit conditions. Figure 12 shows the measurement configuration. The impedance characteristics for the conditions, when the ECAs were short circuited by a bare finger and a gloved finger, were derived using a Cole-Cole equivalent circuit.

The Impedance characteristics were determined by applying an ac signal to the sample (between the dual ECA switch) and using a DSP based phase sensitive

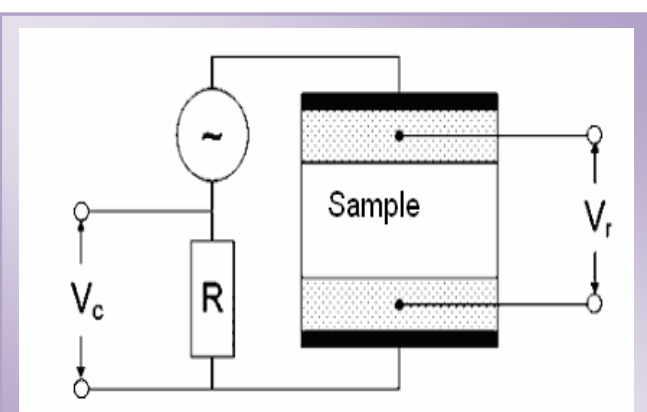

Figure 12: Impedance characteristics measurement configuration detection technique. The excitation current was produced in an impedance spectroscopy system using waveform synthesis. The voltage measurement process was performed synchronously to obtain in-phase and quadrature components of the measured voltage; this required a reference waveform from the waveform synthesizer. The results are shown in Figures 13 and 14.

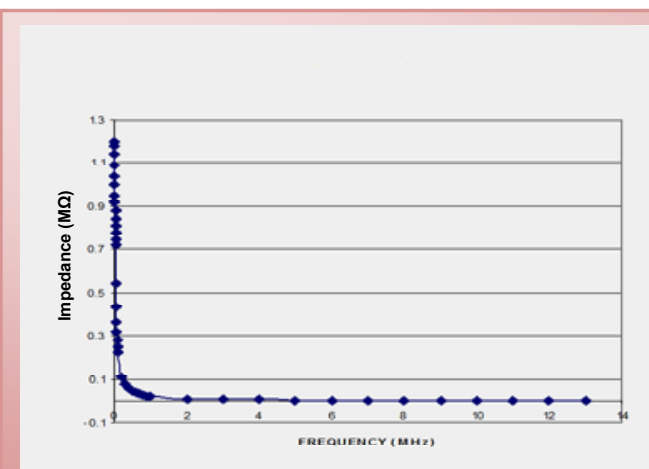

Figure 13: Impedance characteristics of dual ECA switch when it is in open state.

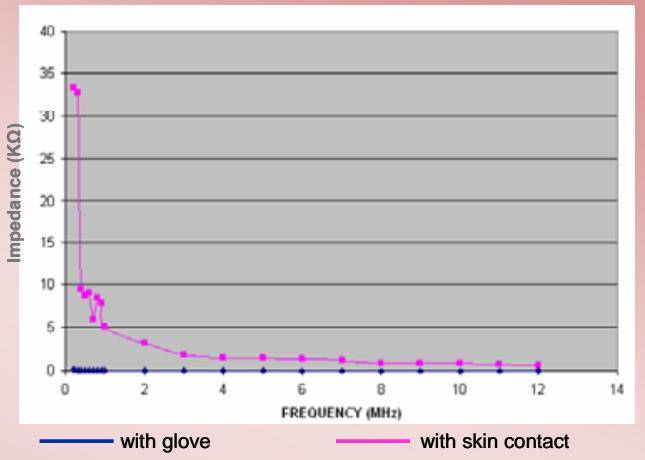

Figure 14: Impedance characteristics of dual ECA switch when it is closed.

Several knitted EAT structures have been developed in the University of Manchester, and two products, i.e. a sensing EAT and an active EAT, were introduced in this paper. The fundamental principal of the above demonstrated technology is based on localising electro-conductive fibres within the conventional fibres of a textile structure. Although the technology has the ability to develop textile sensors and actuators one of its draw backs is the limitation in the development of intelligent and truly smart textile structures. As such a new line of research was initiated by research group in the University of Manchester; the basic concept is to embed electronic chips within the fibres of a yarn. The background to the research is given below.

The underlying core technology for clothing manufacture is performed in phases, with the most significant processing steps summarised below:

- Fibre assembling process: During this step a multitude of fibres are assembled to form a yarn. The most important characteristic of a yarn is that it has only a length and a thickness (its thickness can be neglected when compared to its almost unlimited length), and its flexibility. Generally yarns used in making 
clothes are made either from natural, man-made fibres, or from a blend of natural and man-made fibres.

- Fabric manufacturing process: In this step the yarns are assembled into a fabric, in order to create a textile fabric. Unlike a yarn, a textile fabric has a length, a width and a thickness (again the thickness of a fabric can be neglected against its length and width); also a textile fabric can be used to cover a two-dimensional surface. Like a yarn, a textile fabric is a flexible structure, which is a very important characteristic for making a comfortable garment. The most important yarn assembling techniques are the weaving and knitting processes. In weaving two parallel sets of yarns are interlaced orthogonally, whereby in knitting only one set of yarns can be interlooped to form a fabric structure.

- Fabric colouration and finishing process: In this step a fabric structure is given colour and/or better handling performance. Although fibres undergo only a physical change in the first two processes, in this step fibres would be exposed to chemical reactions.

- Fabric assembling process: In this step the textile fabric is cut into shaped panels and sewn together to form a three-dimensional shell (i.e. a garment).

In all the processes mentioned above, the fibres will be subjected to tensile, compressive, bending and torsional deformations, and during the fabric colouration and finishing process the fibres will be subjected to higher temperatures and pressures. On the other hand the fibres will be subjected to similar deformations during the normal use of a garment, and its washing.

The objective of our work is to integrate the electronically functional chips (referred to as the chip in further text) with textile fibres in a manner that it is protected from the negative influences outlined previously. This objective could be achieved by either inserting the chip into a textile fibre or encapsulating the chip within a bundle of fibres, the two techniques are discussed briefly below.

Inserting the chip into a textile fibre: this could be achieved with only man-made fibres by inserting the chip during the extrusion of the fibres. Although this technique could protect the chip from the tensile and bending stresses and the temperature, pressure and other chemical stresses the textile fibre will be subjected to during processing; this method, however, will not protect the chip from the torsional deformations of the textile fibre.

Encapsulation of the chip within a bundle of fibres: this method can protect the chip from all the aforementioned deformations and stresses, and it is limited not only to man-made fibres. Therefore, our aim was to create the science base to package the chip within a fibre bundle. The research objectives were:

- The development of the encapsulation technique;

- The modelling of the encapsulation process;

- The evaluation of the fibre encapsulated chip, i.e. for mechanical stresses etc.;

- The development of a continuous process for encapsulating the chips;

- The development of a technique of connecting the chip with metal core filament fibres. 
As mentioned earlier our concept is to package the chip inside a bundle of fibres in a manner that it is protected from all forms of mechanical, thermal and pressure forces. Our solution was to position the chip inside a bundle of man-made filament fibres, and then to encapsulate the area with a polymer matrix. In the following text the following definitions will be used:

- The chip encapsulated area as the Encapsulated Area (EA); and

- The resultant yarn as the Electronically Functional Yarn (EFY).

The EAs can be positioned at regular intervals along the length of the filament fibres as shown in the Figure 15. This concept would enable the EFY to be processed in weaving, knitting and sewing processes.

Figure 15: Schematic diagram of a section of EFY

\section{$\underline{\text { Resin Impregnated EA Process }}$}

This technique has the advantage that all types of deformations and stresses, which the EFY would be subjected during the textile processing, the day to day wearing of the garment (with the EFY) and its washing and ironing etc., can be endured by the filament fibres and the polymer resin. The polymer resin would also insulate (seal-off) the chip from liquids and dust etc. The described concept will also enable fine metal wire (possibly in the form

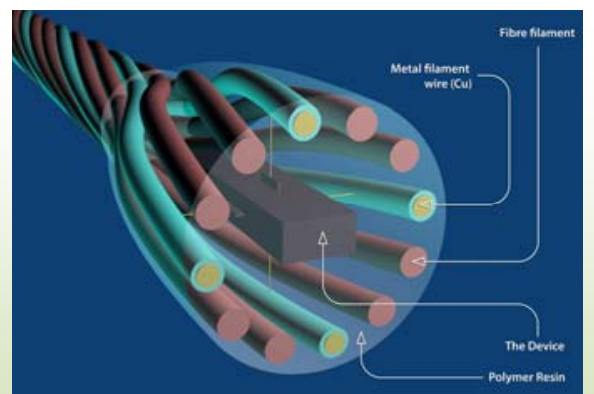

Figure 16: Cross sectional view of an encapsulated area of a polymeric monofilament with a $\mathrm{Cu}$ or silver wire core) to be incorporated into the EFY for powering the chip and signal transmission (Figure 16). The reinforcing of the filament fibres and the fine metal wire, and the sealing-off of the chips is a major challenge to the production of EA with properties that are adequate for further processing. A necessary prerequisite for the successful production of EFY was the use of computer simulation, along with static and dynamic modelling of the process for making the EA. As the EA consists of several phases; i.e. a discontinuous polymer resin, continuous polymer filament fibres, copper cored polymer filament fibres, and, therefore, the EA can be considered as a composite system. The matrix phase would bind the filament fibres together and hermetically envelop the chip, thus providing the composite system (EA) phase unity, shape and size stability. The process of fabrication depended on the physical and the chemical characteristics of the resin, especially on the curing properties. The curing was achieved at room temperature; however, the curing time has to be optimised.

Based on the mathematical modeling an experimental rig was designed and developed to encapsulate micro chips within filament fibres, and different chips were encapsulated to produce EFY; two examples are given below. 
Figure 17: RFID fibre (PE fibre embedded with Hitachi MU tag)

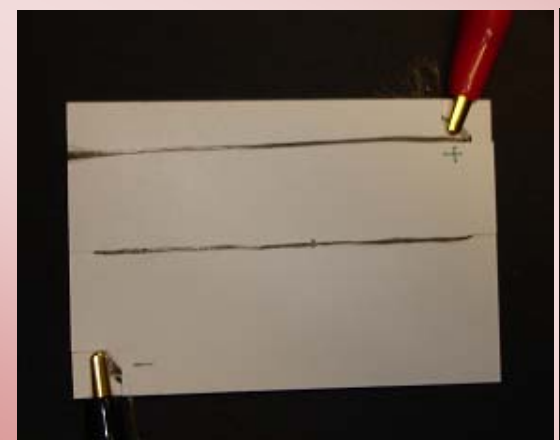

LED not activated

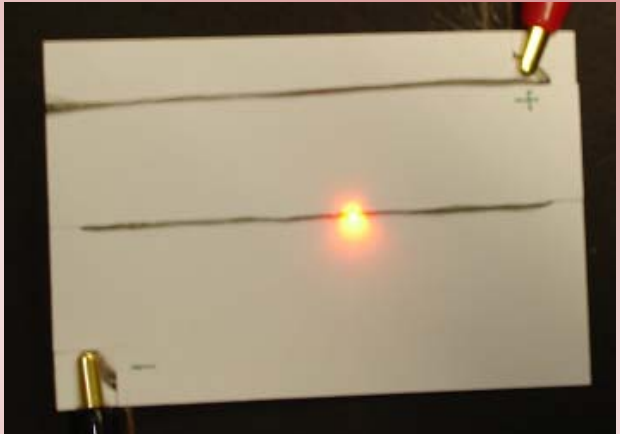

LED activated

Figure 18: Yarn with LED

\section{References}

EAT $^{\circledR}$ (Electrically Active Textiles) is a registered trade mark of Fibre Devices Ltd, UK

Some contents of the paper are based on the following publications:

Wijesiriwardana, R., Dias, T., and Mitcham, K., Fibre-meshed transducers based real time wearable physiological information monitoring system, $8^{\text {th }}$ International symposium on wearable computer proceedings, 40-47 2004

Wijesiriwardana, R., Dias, T. and Mukhopadhyay, S., Resistive fibre-meshed transducers, $7^{\text {th }}$ International symposium on wearable computer proceedings, 2002092003

Wijesiriwardana R., Dias T., Mitcham K. and Hurley, W., Capacitive fiber-meshed transducers for touch and proximity-sensing applications, IEEE Sensor Journal, Vol. 5 Issue 3, (2005)

Wijesiriwardana R., Novel knitted fibre-meshed transducers, PhD Thesis, The University of Manchester, January 2005 